\title{
Spin symmetry breaking and entropy production during the evolution of spinor Bose-Einstein condensate driven by coherent atom beam
}

\author{
Yixin $\mathrm{Xu},{ }^{1}$ Zhongda Zeng, ${ }^{1}$ Zbigniew Domanski, ${ }^{2}$ and Zhibing $\mathrm{Li}$ (1) $1,3,4,{ }^{*}$ \\ ${ }^{1}$ School of Physics, Sun Yat-Sen University, Guangzhou, 510275, People's Republic of China \\ ${ }^{2}$ Institute of Mathematics, Czestochowa University of Technology, 42-201 Czestochowa, Poland \\ ${ }^{3}$ State Key Laboratory of Optoelectronic Materials and Technologies, Guangzhou, 510275, People's Republic of China \\ ${ }^{4}$ Guangdong Province Key Laboratory of Display Material and Technology, Guangzhou, 510275, People's Republic of China
}

(Received 27 September 2019; accepted 18 March 2020; published 10 April 2020)

\begin{abstract}
The spinor condensate with spin states degenerated in the ground spin-space provides a unique platform for investigating the edge of quantum mechanics and statistical physics. We study the evolution of the condensate under the scattering of a coherent atom beam. The time-dependent magnetization, entanglement entropy, statistical entropy, and the entropy production rate are calculated. A novel spontaneous spin symmetry breaking is found during the evolution. It is shown that the stationary spin distribution can be controlled by the coherent spin state of the incident atom beam, therefore the atom-condensate scattering provides a new way to generate designable spin distributions of the condensate.
\end{abstract}

DOI: 10.1103/PhysRevResearch.2.023028

Statistical behavior of quantum systems with few degrees of freedom would exhibit subtle interplay between quantum statistics and quantum mechanics. In recent years, a number of ingenious experiments on the thermalization of small quantum systems have been carried out [1-4]. They revealed that the quantum entanglement is indispensable for validating the eigenstate thermalization hypothesis of equivalence between the thermal ensemble averages and the quantum-mechanical time averages of observable quantities in isolated quantum systems that were initialized in coherent superpositions of eigenstates [5-7]. Recently, the entropy production rate at the steady state of a Bose-Einstein condensate (BEC) coupled with a micromechanical resonator has been measured and the irreversibility of the system has been discussed [8]. On the other hand, the BEC is an example of spontaneous symmetry breaking (SSB), where the gauge symmetry is broken below the critical temperature. For a BEC having spin degrees of freedom, there would be another type of SSB in the degenerated space of spin-states with the lowest energy. Any new mechanism for SSB is of broad interest for physics. The present paper is interested in the microdistribution of the BEC with spin degrees of freedom and the SSB that is not temperature-induced but driven by a coherent beam of atoms. In the process, the entropy of the BEC can be transferred to the current of atoms through the quantum entanglement between the propagating atoms and the BEC when they interact. We demonstrate that (i) a symmetric incident beam can drive

\footnotetext{
*Corresponding author: stslzb@mail.sysu.edu.cn
}

Published by the American Physical Society under the terms of the Creative Commons Attribution 4.0 International license. Further distribution of this work must maintain attribution to the author(s) and the published article's title, journal citation, and DOI. the BEC to the SSB phase and the entropy production rate attains its maximum at the instance when the magnetization as the indication of broken spin up-down symmetry becomes nonzero; and (ii) the steady spin distributions of the BEC are dictated by the incident atom beams which are controllable.

The BECs composed of atoms with spin and referred to as spinor BECs (SBECs) possess large internal degrees of freedom [9-12]. Spin-mixing experiments have confirmed that the spin states of SBECs could remain coherent for seconds [13-16]. The SBEC degenerated in magnetic polarizations has finite entropy even when its spatial mode is frozen. A rich variety of phases that rely on inter-particle interactions and interactions with external fields have been predicted [17-20]. Properties of the ground state can be changed dramatically by the Feshbach resonance [21-25]. Near the resonance, the magnitude of scattering length of two atoms diverges. For the atom-condensate scattering, additionally, the scattering length is enhanced by a factor proportional to the number of atoms due to their coherence in the condensate [26]. Since the condensate may consist of thousands(and more) of atoms, the atom-condensate scattering should have a non-negligible probability and thus would be experimentally feasible.

We assume that the spin state of the incident atoms is a pure quantum state, hence the input entropy is zero. On the other hand, the outgoing atom is entangled with the condensate, forming a finite entanglement entropy. Therefore the atom beam contributes a negative entropy current to the condensate as long as its state is time-dependent. We further assume that the incident atoms have very low kinetic energy and will thus neither excite spatial modes of the condensate nor lead to heating and/or atom loss of the condensate. It requires temperatures of the condensate and the source of incident beam lower than $\hbar \omega \sim n K$ for a typical optical trap of $\omega=100 \mathrm{~Hz}$. Thereby, the entropy associated with the entanglement of the condensate and scattered atom originates from spin exchange 
which in turn relies crucially on the principle of identical particles in quantum mechanics and internal symmetries of the system. This entropy appears even if the spin-dependent interaction is negligible during the scattering process.

Two major mechanisms for entropy production in the condensate involve two characteristic times: the scattering time of a propagating atom $t_{s}$ and the decoherence time $t_{d}$. An atom emitted from a source BEC of temperature $T$ will travel a distance of its thermal wave length in a time of $\frac{\pi \hbar}{k_{B} T}$, which should be of the order of $t_{s}$. In the scattering, the outgoing atom shares some information of the condensate and entangles with the latter. The entanglement entropy $S_{e}$ is produced at the same time. On the other hand, there exists an unavoidable weak interaction between the condensate and environment, for instance through the residual magnetic field or an instability of the optical trap that could cause decoherence of the condensate. The time $t_{d}$ is the mean time that the decoherence would happen, accomplishing the statistical entropy $S_{s t}$. We will suppose that $t_{d}<t_{s}$. This means that the condensate is always decoherent after each scattering.

The SBEC consisting of $N$ atoms, each having spin $f=1$, is confined in an optical trap. We adopt the single mode approximation [15,27-30]. In the absence of an external magnetic field, the energies are proportional to $s(s+1)$ with $s$ being the spin of the condensate. We further assume that the lowest energy level has maximum spin $s=N$ and is degenerate in magnetic polarizations. This is the case of the dilute atom gas with ferromagnetic spin-spin interaction, such as that of ${ }^{87} \mathrm{Rb}$. In principle, the spin of the condensate may change from $s=N$ to $N-2$ in the scattering. However, the scattering branch ratios of channels between $s=N-2$ and $s=N$ are suppressed by a factor $\frac{1}{N}$ and therefore these channels can be neglected when $N$ is large [26]. In other words, the energy of the condensate does not change in the scattering.

Since the coupling strength of spin-independent interaction is much larger than that of the spin-dependent interaction $[19,20]$, we neglect the latter in the atom-condensate scattering. Denote by $a_{\mu}^{+}$and $a_{\mu}\left(c_{\mu}^{+}\right.$and $\left.c_{\mu}\right)$ the creation and annihilation operators of atom in the propagating mode (condensation mode) with spin polarization $\mu$. The Hamiltonian related to the scattering is given by (Appendix A)

$$
H_{s c}=g_{0} \sum_{\mu_{1} \mu_{2}} a_{\mu_{1}}^{+} c_{\mu_{2}}^{+}\left(a_{\mu_{1}} c_{\mu_{2}}+a_{\mu_{2}} c_{\mu_{1}}\right) .
$$

The spin-independent coupling $g_{0}$, which has absorbed the integral of the spatial modes, could be varied by the technique of Feshbach resonance. The second term in the bracket of (1) responsible for the spin-flip is originated from the effect of identical particles.

The scattering amplitudes can be calculated in the Born approximation as the interaction is short ranged. The spin degrees of freedom, however, need to be treated rigorously because the spin symmetry is crucial in our problem. For this reason, we apply the method of fractional parentage coefficients enabling us to go beyond the mean-field approximation $[26,31]$.

In the proposed experiment, all incident atoms come from the same pure state $\left|\xi^{i}\right\rangle_{a}=\sum_{v=-1}^{1} \xi_{v}^{i} a_{v}^{+}|0\rangle_{a}$, with the subscript $a$ labeling the spin states of the propagating atom.
The state of the condensate scattered from an arbitrary spin distribution can be derived using the scattering from base-state vectors of the condensate. A base-state vector of the condensate may be chosen as the eigenstate of the $Z$ component of the spin, $|m\rangle_{c}$, which is uniquely specified by the magnetization quantum number $m$ ranging from $-N$ to $N$. The composite system initially in $\left|m_{i}\right\rangle_{c}\left|\xi^{i}\right\rangle_{a}$ will be scattered to

$$
\left|\Psi_{\xi^{i}}^{m_{i}}\right\rangle=\sum_{m=-N}^{N} \sum_{\mu=-1}^{1} T_{m, \mu}^{m_{i}}|m\rangle_{c}|\mu\rangle_{a} .
$$

The transition matrix $T_{m, \mu}^{m_{i}}$ is given by (Appendix B)

$$
T_{m, \mu}^{m_{i}}=\left(\delta_{m, m_{i}}+C_{N, m ; m_{i}-\mu, \nu}^{N-1,1} C_{N, m_{i} ; m_{i}-\mu, \mu}^{N-1,1}\right) \xi_{v}^{i} .
$$

Two C factors being the Clebsch-Gordan coefficients come from the matrix element $\left\langle m\left|c_{\nu}^{+} c_{\mu}\right| m_{i}\right\rangle$, and $\nu=m-m_{i}+\mu$ due to the conservation of the total spin polarization.

Generally, the condensate is not necessarily in a pure state and should be described by a spin state operator. Denote the spin state operator of the condensate after the $n$th scattering as $\rho_{c}\left(t_{n}\right)$. Thus the initial state of the condensate prior to the $n$th scattering is $\rho_{c}\left(t_{n-1}\right)$. Since $t_{d}<t_{s}$ has been assumed, $\rho_{c}\left(t_{n}\right)$ is diagonal in the representation of magnetization. Let its diagonal elements be $\lambda_{m}\left(t_{n}\right)$. When the outgoing atom is still entangled with the condensate, i.e., for $t_{n-1}<t<t_{d}$, the scattered atom-condensate system is described by the composite spin state operator $\rho(t)=\sum_{m_{i}} \lambda_{m_{i}}\left(t_{n-1}\right)\left|\Psi_{\xi^{i}}^{m_{i}}\right\rangle\left\langle\Psi_{\xi^{i}}^{m_{i}}\right|$. The off-diagonal elements of $\rho(t)$ represent the interference of states with different magnetizations. In this period, the local properties of the condensate are described by the reduced spin operator $\rho_{c}^{\prime}(t)=\operatorname{Tr}_{a} \rho(t)$ where the trace is over the spin space of the propagating atom. The state $\rho_{c}^{\prime}(t)$ would be partially coherent. After $t_{d}$, but before the subsequent scattering, a tiny residue external field will destroy the interference and thus the spin state of condensate will be described by $\rho_{c}\left(t_{n}\right)$, which is $\rho_{c}^{\prime}(t)$ but with all off-diagonal elements set to zero.

Using (2), one can derive the iterative equation

$$
\lambda_{m}\left(t_{n}\right)=\frac{1}{Z\left(t_{n}\right)} \sum_{m^{\prime}} \sum_{\mu=-1}^{1}\left|T_{m, \mu}^{m^{\prime}}\right|^{2} \lambda_{m^{\prime}}\left(t_{n-1}\right),
$$

where $Z\left(t_{n}\right)$ preserves normalization $\sum_{m} \lambda_{m}\left(t_{n}\right)=1$.

The reduced state operator of outgoing atom $\rho_{a}\left(t_{n}\right)$ is derived by tracing out the spin states of the condensate

$$
\rho_{a}\left(t_{n}\right)=\frac{1}{Z\left(t_{n}\right)} \sum_{m_{i}} T_{m, \mu}^{m_{i}} T_{m, \mu^{\prime}}^{m_{i} *} \lambda_{m_{i}}\left(t_{n-1}\right)|\mu\rangle_{a} a\left\langle\mu^{\prime}\right|,
$$

where summations over double repeated indexes $m, \mu, \mu^{\prime}$ are implied. The time-dependent entanglement entropy can be obtained through $\rho_{a}\left(t_{n}\right)$ as $S_{e}\left(t_{n}\right)=-\operatorname{Tr}\left[\rho_{a}\left(t_{n}\right) \ln \rho_{a}\left(t_{n}\right)\right]$. It is transferred to the environment when the outgoing atom is "detected" by the environment. On the other hand, the decoherence appears within $t_{d}$ and leads to the statistical entropy, $S_{s t}\left(t_{n}\right)=-\sum_{m} \lambda_{m}\left(t_{n}\right) \ln \lambda_{m}\left(t_{n}\right)$. The entropy production rate is the total entropy produced in the $n$th scattering, $S_{p}\left(t_{n}\right)=$ $S_{e}\left(t_{n}\right)+S_{s t}\left(t_{n}\right)-S_{s t}\left(t_{n-1}\right)$. After a sufficiently long time, the condensate is driven to a steady state characterized by a 


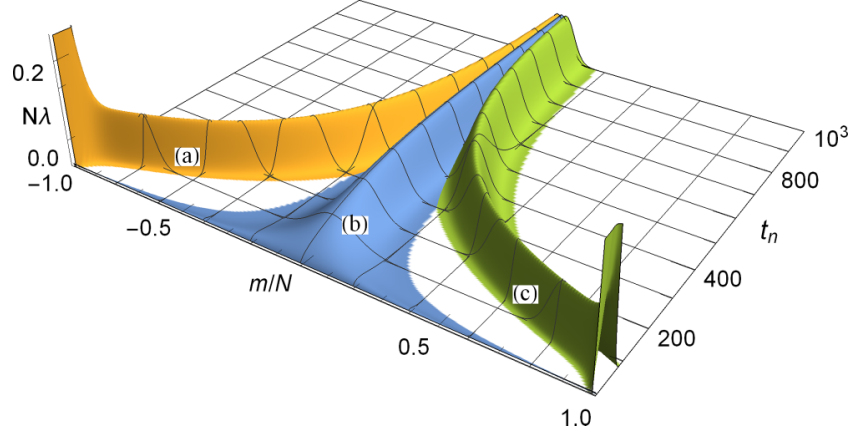

FIG. 1. The time-dependent spin distributions of spin-1 condensate from initial distributions: (a) $\lambda_{m}(0)=\delta_{m,-N}$, (b) $\frac{1}{2 N+1}$, and (c) $\delta_{m, N}$, respectively. The condensate is scattered by the same species of atoms with incident state $|0\rangle_{a}$. The axes $m / N$ is the magnetization quantum number per atom. The time $t_{n}=n$ is in the unit of times of scattering. Here, $N=100$ is used for an instance.

constant statistical entropy, whereas the entropy production rate converges to a constant entanglement entropy.

Let us first specify the incident state to $\left|\xi^{0}\right\rangle_{a}=|0\rangle_{a}$ and compare the evolutions of three exemplary initial states of the condensate: (a) $\lambda_{m}(0)=\delta_{m,-N}$ (the negative fully ordered distribution), (b) $\frac{1}{2 N+1}$ (the microcanonical distribution), and (c) $\delta_{m, N}$ (the positive fully-ordered distribution). The timedependent spin distributions from the three initial states are plotted in Fig. 1. We have scaled $\lambda_{m}$ and $m$ by $N$ and $1 / N$, respectively, therefore the shape of plot for any large enough $N$ is basically the same as the present example $(N=100)$ if the time axis is scaled by $1 / N$ too. The reason for using the number of times of scattering as time but not the physical time is to make the plot independent the intensity of the incident beam. The physical time of a scattering, $t_{s}$, is shorter/longer for a stronger/weaker incident beam. However, the thermal wavelength of incident atoms should be shorter than the distance between two atoms incoming in succession. For an atom source of temperature $T \sim n K$, the condition is fulfilled when $t_{s}>0.02$ second.

After a sufficiently long time, all distributions converge to steady distributions that are independent of the initial spin state of the condensate but completely determined by the incident atom states. The steady spin distributions for incident states $|\xi\rangle_{a}=\frac{1}{\sqrt{2}}\left(|0\rangle_{a}+\cos \theta|-1\rangle_{a}+\sin \theta|1\rangle_{a}\right)$ are presented in Fig. 2 from left to right for $\theta=0, \frac{\pi}{6}, \frac{\pi}{4}, \frac{\pi}{3}$, and $\frac{\pi}{2}$. It is clearly seen that these distributions are neither uniform nor Boltzmann-like distributions. The steady spin distributions for the middle three $\theta$ 's appear to be Gaussianlike with the half-width approximately proportional to $\frac{1}{\sqrt{N}}$, see the inset of Fig. 2.

The system has a spin-reversal symmetry and we will show that this symmetry can be broken in the course of the evolution. Consider the condensate having the symmetric initial spin distribution $\lambda_{m}(0)=\delta_{m, 0}$ and the incident-atom state $\left|\xi^{0 y}\right\rangle_{a}=\frac{1}{\sqrt{2}}\left(|-1\rangle_{a}+|1\rangle_{a}\right)$. The state $\left|\xi^{0 y}\right\rangle_{a}$ is an eigenstate of the $Y$ component of the atom spin with vanishing projection on the $Y$ direction. The composite system still has the spin-reversal symmetry. The evolving distribution of $\left\{\lambda_{m}\left(t_{n}\right)\right\}$ is shown as the upper panel of Fig. 3. A bifurcation

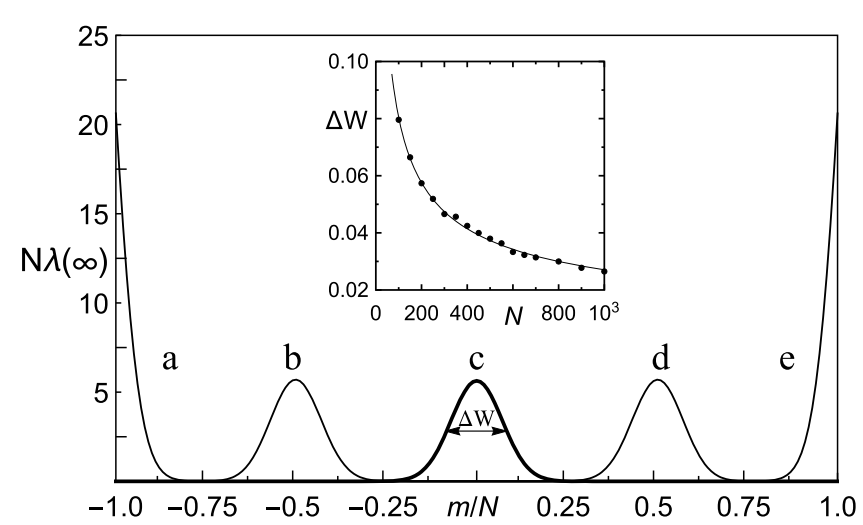

FIG. 2. The steady spin distributions for spin-1 condensate of $N=100$ spinors under the scattering of the atom beam with incident states $\frac{1}{\sqrt{2}}\left(|0\rangle_{a}+\cos \theta|-1\rangle_{a}+\sin \theta|1\rangle_{a}\right)$. Curves (a), (b), (c), (d), and (e) correspond to $\theta=0, \frac{\pi}{6}, \frac{\pi}{4}, \frac{\pi}{3}$, and $\frac{\pi}{2}$, respectively. The inset is the half-width of the distribution (c) for $N \leqslant 10^{3}$.

emerges suddenly at a time of the evolution, indicating the spin-reversal symmetry breaking. A hint to the symmetry breaking is given by the time-dependent fluctuation of magnetization quantum number per atom, $\chi(t)=\sum_{m}\left(\frac{m}{N}\right)^{2} \lambda_{m}(t)$,

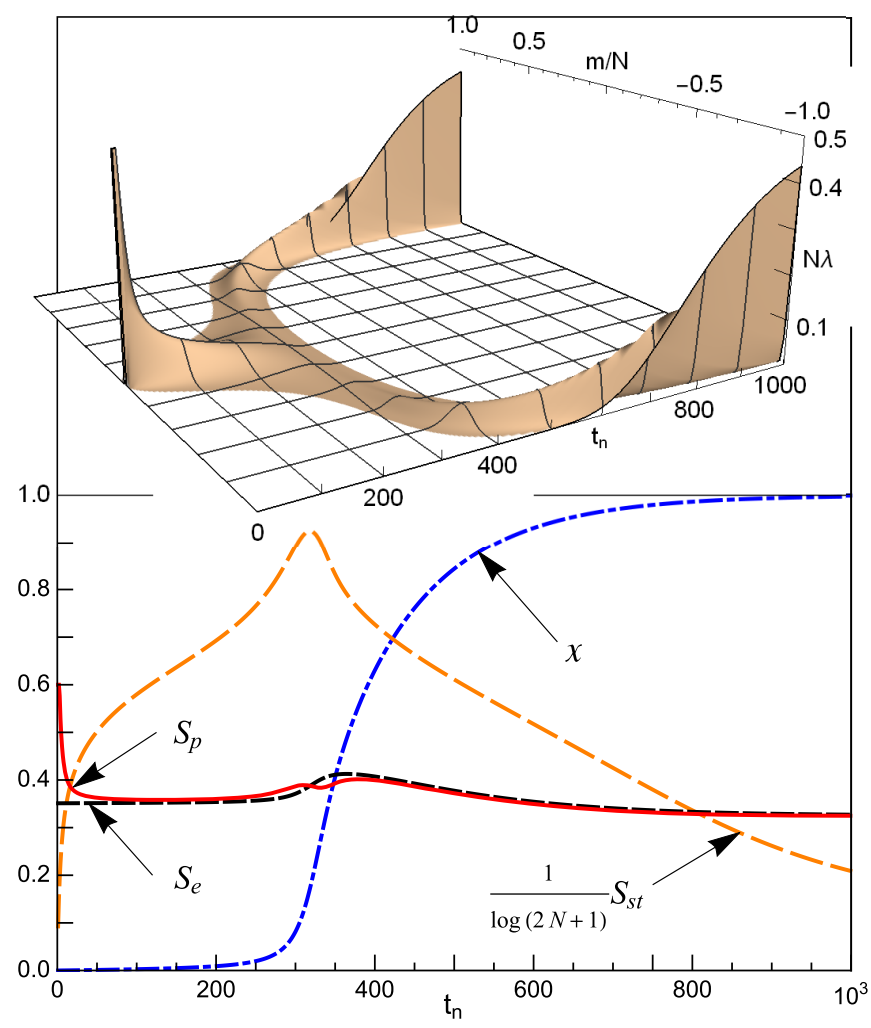

FIG. 3. The spin-1 condensate of $N=100$ is scattered from the nonpolarized initial spin distribution $\lambda_{m}(0)=\delta_{m, 0}$ by the same species of atoms in the incident state $\left|\xi^{0 y}\right\rangle_{a}=\frac{1}{\sqrt{2}}\left(|-1\rangle_{a}+|1\rangle_{a}\right)$. In the lower panel, the curves are the time-dependent magnetization fluctuation (blue dash-dotted), the statistical entropy divided by $\ln (2 N+1)$ (orange long-dashed), the entanglement entropy (black short-dashed), and the entropy production rate (red solid). The upper panel is the time-dependent spin distribution. 
presented in the lower panel of FIG. 3 as the blue-dashdotted line. At a mesoscopically short time $t_{0}=\tau N$, with a finite $\tau \ll 1$, the process resembles a random walk on $\left\{x_{m}=\frac{m}{N}: m=-N, \ldots, N\right\}$, therefore the spin distribution is a Gaussian-like centered at zero and cumulants of $m / N$ of order higher than two vanish. In the large $N$ limit, we obtain $\chi(t) \sim \chi\left(t_{0}\right)\left[1-\frac{6}{7} \chi\left(t_{0}\right)\left(t-t_{0}\right)\right]^{-1}$ for $t>t_{0}$ (Appendix C). Since $\chi\left(t_{0}\right)=\frac{2 D t_{0}}{N^{2}}=\frac{2 D \tau}{N}$, with $D$ the random-walk diffusion coefficient, then $\chi(t) \sim \frac{2 D \tau}{N}\left[1-\frac{12 D \tau}{7 N}\left(t-t_{0}\right)\right]^{-1}$ is tiny for a large $N$ but it blows up at $t \sim N$. The diverging fluctuation invalidates the Gaussian-like assumption and triggers the bifurcation.

The time-dependent statistical entropy (orange longdashed line, with the magnitude scaled by $[\ln (2 N+1)]^{-1}$, the entanglement entropy(black short-dashed line), and the total entropy production rate(red solid line) are also plotted in FIG. 3. Near the bifurcation time, $\chi(t)$ grows rapidly and $S_{s t}$ as well as $S_{e}$ attain maxima. After a sufficiently long time, the condensate will choose one of two fullyordered states by incident, hence the spin-reversal symmetry is broken. None of the entropies is a monotonic function of time. In the steady state, $S_{s t}$ does not change and hence $S_{p}=S_{e}$.

Summary and discussion. We have investigated the evolution of the spinor Bose-Einstein condensate that is scattered by atoms from a coherent atom source. The dynamics is solely governed by the symmetry of the internal degrees of freedom. We obtained the time-dependent: spin distribution of the condensate, magnetization, magnetization fluctuation, statistical and entanglement entropies, and total entropy production rate. It is found that the steady states of the condensate do not depend on the initial state of the condensate but are determined by the incident atom state. This provides a possible way to prepare various spin distributions of the condensate, which are neither uniform nor Boltzmann-like distributions, by choosing suitable incident states. Designable spin distributions are useful for studying spin dynamics of condensates. A novel spontaneous spin symmetry breaking phenomenon is found in the condensate evolution with the initial magnetization quantum number being zero and the incident atom state being a symmetric coherent superposition of $|-1\rangle_{a}$ and $|1\rangle_{a}$. The phase-transition-like symmetry breaking accompanied with the bifurcation of spin distribution emerges at a mesoscopic time of about twice the atom number of the condensate. After that time, the SBEC is driven to the polar phase whose spin distribution develops into two ordered states with opposite magnetization. Each experiment will choose one out of these two possible ordered states. The results would add new insight to spontaneous symmetry breaking and entropy production in small quantum systems. To derive the above results, we have assumed that the temperature of the condensate is lower than the excitation energy and the incident atoms have very small kinetic energy so that the condensate remains in the degenerate space of ground states. The required sub-nano kelvin temperature would be realized in the future. The other less strict assumption is that prior to each scattering the condensate is decoherent, i.e., $t_{d}<t_{s}$. Our study can be generalized to the reverse case of $t_{d}>t_{s}$.
Acknowledgments. Z.D. thanks the hospitality of the Sun Yat-sen University. The project is supported by the KeyArea Research and Development Program of Guangdong Province (Grant No. 2019B030330001) and the National Key Research and Development Project of China (Grant No. 2016YFA0202001).

\section{APPENDIX A: THE INTERACTION HAMILTONIAN FOR THE SCATTERING}

Take the BEC of spin-1 bosons ${ }^{87} \mathrm{Rb}$ scattered by ${ }^{87} \mathrm{Rb}$ atoms as an example. The scattering of two identical ${ }^{87} \mathrm{Rb}$ atoms has two channels related to the total coupled $\operatorname{spin} F=0$ and $F=2$. Correspondingly, the effective Hamiltonian of this two-body interaction can be decomposed into two terms [17],

$$
H_{\mathrm{int}}=2 \pi a_{0} \int d \mathbf{r} P^{(0)}(\mathbf{r})+2 \pi a_{2} \int d \mathbf{r} P^{(2)}(\mathbf{r}),
$$

where the operator $P^{(F)}$ projects two-particle states onto states of total hyperfine spin $F$ and $a_{F}$ is the s-wave scattering length for the $F$ channel. The interaction conserves both the total spin and its $Z$-component. For ${ }^{87} \mathrm{Rb}$ atoms, the interaction (A1) is ferromagnetic and the scattering lengths $a_{0}, a_{F}$ are almost equal with negative $a_{2}-a_{0}=-1.5(3)$ in units of the Bohr radius [14,32]. Let $\psi_{\mu}^{+}(\mathbf{r})\left(\psi_{\mu}(\mathbf{r})\right)$ be the quantum field creation (annihilation) operator of atom with spin $Z$ component at a position $\mathbf{r}$. The projection operators can thus be written as [19]

$$
P^{(F)}(\mathbf{r})=P_{\mu_{1}, v_{1} ; \mu_{2}, \nu_{2}}^{(F)} \psi_{\mu_{1}}^{+}(\mathbf{r}) \psi_{\nu_{1}}^{+}(\mathbf{r}) \psi_{\mu_{2}}(\mathbf{r}) \psi_{\nu_{2}}(\mathbf{r}),
$$

where

$$
P_{\mu_{1} \nu_{1}, \mu_{2} \nu_{2}}^{(F)}=\sum_{m=-F}^{m=F} C_{F, m ; \mu_{1}, \nu_{1}}^{1,1} C_{F, m ; \mu_{2}, \nu_{2}}^{1,1}
$$

and $C_{F, m ; \mu, v}^{1,1}$ are the Clebsch-Gordan coefficients.

Because the spin-spin coupling is ferromagnetic, the ground states of the condensate have total spin $N$ that is equal to the number of atoms in the condensate. We denote as $\varphi(\mathbf{r})$ the spatial mode of a single particle in the ground state. This includes the effects of atom-atom interaction and a spherical trapping potential. In the single mode approximation, $\varphi(\mathbf{r})$ does not depend on the spin polarization and is normalized $\int|\varphi|^{2} d \mathbf{r}=1$. Let the single-particle mode of the condensate be created by $c_{\mu}^{+}$and the corresponding annihilation operator be $c_{\mu}$. In the coordinate representation

$$
\left\langle\mathbf{r}, \mu^{\prime}\left|c_{\mu}^{+}\right| 0\right\rangle=\varphi(\mathbf{r}) \delta_{\mu^{\prime} \mu} .
$$

On the other hand, the propagating states of a single particle with wave vector $\mathbf{k}$ are created by $a_{\mu}^{+}(\mathbf{k})$. In the coordinate representation,

$$
\left\langle\mathbf{r}, \mu^{\prime}\left|a_{\mu}^{+}(\mathbf{k})\right| 0\right\rangle=\phi_{\mathbf{k}}(\mathbf{r}) \delta_{\mu^{\prime} \mu} .
$$

The field operators can be expanded in the mode operators as

$$
\begin{gathered}
\psi_{\mu}^{+}(\mathbf{r})=\varphi(\mathbf{r}) c_{\mu}^{+}+\sum_{\mathbf{k}} \phi_{\mathbf{k}}^{*}(\mathbf{r}) a_{\mu}^{+}(\mathbf{k})+\cdots \\
\psi_{\mu}(\mathbf{r})=\varphi(\mathbf{r}) c_{\mu}+\sum_{\mathbf{k}} \phi_{\mathbf{k}}(\mathbf{r}) a_{\mu}(\mathbf{k})+\cdots
\end{gathered}
$$


where "..." stands for terms related to other condensed modes which can be neglected as the condensate remains in the space of the ground states. Substitution of (A6) and (A7) into (A2) and then in the Eq. (A1) leads to the interaction Hamiltonian responsible for the scattering

$$
H_{s c}=\sum_{\mathbf{k}, \mathbf{k}^{\prime}} g_{F}\left(\mathbf{k}, \mathbf{k}^{\prime}\right) c_{\mu_{1}}^{+} a_{\nu_{1}}^{+}(\mathbf{k}) P_{\mu_{1} \nu_{1}, \mu_{2} \nu_{2}}^{(F)} a_{\nu_{2}}\left(\mathbf{k}^{\prime}\right) c_{\mu_{2}},
$$

where

$$
g_{F}\left(\mathbf{k}, \mathbf{k}^{\prime}\right)=8 \pi a_{F} \int d \mathbf{r} \phi_{\mathbf{k}}^{*}(\mathbf{r}) \phi_{\mathbf{k}^{\prime}}(\mathbf{r})|\varphi(\mathbf{r})|^{2} .
$$

Summations over $F=0,2$ and over repeated subscripts have been implied in (A8).

One can adopt the $s$-wave approximation in the low energy and short-range scattering regime. Therefore, the integral in (A9) does not depend on the directions of $\mathbf{k}$ and $\mathbf{k}^{\prime}$. We further assume that the total spin of the condensate is unchanged in the scattering. Hence, the kinetic energy of the propagating atom and the energy of the condensate are conserved separately as the latter depends only on the total spin. As a consequence of the energy conservation we have $k^{\prime}=k$. Therefore only spin-flipping interactions need to be considered and (A8) can be written as

$$
H_{s c} \simeq \sum_{\mathbf{k}, \mathbf{k}^{\prime}} g_{F}(k) c_{\mu_{1}}^{+} a_{\nu_{1}}^{+}(\mathbf{k}) P_{\mu_{1} \nu_{1}, \mu_{2} \nu_{2}}^{(F)} a_{\nu_{2}}\left(\mathbf{k}^{\prime}\right) c_{\mu_{2}} .
$$

In our model, $k=\left|\mathbf{k}^{\prime}\right|$ is determined by the incident beam and all directions of the scattered outgoing wave are equally probable. Therefore, only the spin exchange matters. Neglecting the difference between values of $a_{0}$ and $a_{2}$ in scatterings of the condensate and the propagating atom, and using the explicit expressions for the Clebsch-Gordan coefficients in (A3), we obtain the Hamiltonian for the spin degrees of freedom

$$
H_{s c}=g_{0} \sum_{\mu_{1} \mu_{2}} a_{\mu_{1}}^{+} c_{\mu_{2}}^{+}\left(a_{\mu_{1}} c_{\mu_{2}}+a_{\mu_{2}} c_{\mu_{1}}\right),
$$

which is the equation (1) of the main text.

\section{APPENDIX B: THE TRANSITION MATRIX}

Using two different methods, we derive the transition matrix for the BEC of $N$ spin-1 atoms scattered by the same species of atoms.

\section{Preliminary}

Let $|s, m\rangle_{c}$ denotes the eigenstates of the total spin and its $Z$-component operators. They can be generated by $N$ creation operators of the condensate mode [26]

$$
|s, m\rangle_{c}=\left[\left(c^{+}\right)^{N}\right]_{s, m}|0\rangle,
$$

where the square bracket $[\cdots]_{s, m}$ means to couple the operators in the bracket to the operator that create a fully symmetric normalized state with quantum numbers $(s, m)$. On the other hand, the propagating state of an atom with wave vector $\mathbf{k}$ is created by $a_{\mu}^{+}(\mathbf{k})$ as

$$
|\mathbf{k}\rangle_{a}|\mu\rangle_{a}=a_{\mu}^{+}(\mathbf{k})|0\rangle,
$$

where $\mu$ refers to the spin polarization of the atom. Consider a polarized initial state $|i\rangle=\left|\mathbf{k}_{i}\right\rangle_{a}\left|w_{i}\right\rangle$ with $\left|w_{i}\right\rangle=\left|\mu_{i}\right\rangle_{a}\left|s, m_{i}\right\rangle_{c}$ being the spin state. Assumption that the incident atom has spin one leads to the following scattered final spin of the condensate $s_{\sigma}=s+2 \sigma, \sigma=0, \pm 1$. Denote the final state as $|f\rangle=\left|\mathbf{k}_{\sigma}\right\rangle_{a}\left|w_{\sigma \mu}\right\rangle$ where $\sigma$ and $\mu$ label the spin of the condensate and the spin polarization of the outgoing atom, respectively. Thus, there are nine possible scattering channels that correspond to $\left|w_{\sigma \mu}\right\rangle=|\mu\rangle_{a}\left|s_{\sigma}, m\right\rangle_{c}$ for $\sigma=0, \pm 1$ and $\mu=0, \pm 1$, and $m=m_{i}+\mu_{i}-\mu$ due to the conservation of the spin component.

In the Born approximation, the transition amplitude of spin-flip scattering is given by

$$
A_{\sigma, \mu}^{i}=-\frac{M}{2 \pi \hbar^{2}} T_{m, \mu}^{m_{i}, \mu_{i}},
$$

where the transition matrix reads

$$
T_{m, \mu}^{m_{i}, \mu_{i}}=\left\langle\left. w_{\sigma \mu}\right|_{a}\left\langle\mathbf{k}_{\sigma}\left|H_{s c}\right| \mathbf{k}\right\rangle_{a} \cdot \mid w_{i}\right\rangle
$$

Substitution of $H_{s c}$ in the above equation by the Hamiltonian given in Eq. (1) of the main text leads to

$$
T_{m, \mu}^{m_{i}, \mu_{i}}=g_{0}\left[N \delta_{\sigma, 0} \delta_{m, m_{i}} \delta_{\mu, \mu_{i}}+{ }_{c}\left\langle s_{\sigma}, m\left|c_{\mu_{i}}^{+} c_{\mu}\right| s, m_{i}\right\rangle_{c}\right]
$$

where the spin conservation constraint $m+\mu=m_{i}+\mu_{i}$ is satisfied automatically.

Below, we derive the matrix element ${ }_{c}\left\langle s_{\sigma}, m\left|c_{\mu_{i}}^{+} c_{\mu}\right| s, m_{i}\right\rangle_{c}$ by the method of fractional parentage coefficients (FPCs) [33]. Then, based on the Wigner-Echart theorem, we verify the result for the special case of the SBEC state with maximal total spin $s=N$.

\section{General transition matrix by the FPCs}

The second term in the square bracket of (B5) involves terms that are generated by contractions of $c_{\mu}$ with all the creation operators on its right side (right contractions) as well as terms due to the analogues left contractions of $c_{\mu_{i}}^{+}$. There are $N^{2}$ such terms and all are the same because of the permutation symmetry. Therefore we need to calculate one right and one left contraction. This can be done with the method of FPCs. For instance, to calculate the right contraction we rewrite the normalized operator $\left[\left(c^{+}\right)^{N}\right]_{s m_{i}}$ as $[31,34]$

$$
\begin{aligned}
& {\left[\left(c^{+}\right)^{N}\right]_{s m_{i}}} \\
& =\frac{a_{s}^{\{N\}}}{\sqrt{N}} \sum_{\mu^{\prime}} C_{s, m ; m_{i}-\mu^{\prime}, \mu^{\prime}}^{s+1,1}\left[\left(c^{+}\right)^{N-1}\right]_{s+1, m_{i}-\mu^{\prime}} c_{\mu^{\prime}}^{+} \\
& \quad+\frac{b_{s}^{\{N\}}}{\sqrt{N}} \sum_{\mu^{\prime}} C_{s, m_{i} ; m_{i}-\mu^{\prime}, \mu^{\prime}}^{s-1}\left[\left(c^{+}\right)^{N-1}\right]_{s-1, m_{i}-\mu^{\prime}} c_{\mu^{\prime}}^{+},
\end{aligned}
$$

where the factors

$$
\begin{aligned}
& a_{s}^{\{N\}}=\sqrt{\frac{\left(1+(-1)^{N-s}\right)(N-s)(s+1)}{2 N(2 s+1)},} \\
& b_{s}^{\{N\}}=\sqrt{\frac{\left(1+(-1)^{N-s}\right) s(N+s+1)}{2 N(2 s+1)}},
\end{aligned}
$$

known as the FPCs, were given in Ref. [33]. They vanish when $N-s$ is odd, otherwise $\left(a_{s}^{\{N\}}\right)^{2}+\left(b_{s}^{\{N\}}\right)^{2}=1$. Using 
(B6)-(B8), we obtain the general expression for the matrix elements

$$
\begin{aligned}
& { }_{c}\left\langle s_{\sigma}, m\left|c_{\mu_{i}}^{+} c_{\mu}\right| s, m_{i}\right\rangle_{c} \\
& =\left\langle 0\left|\left[c^{N}\right]_{s_{\sigma} m} c_{\mu_{i}}^{+} c_{\mu}\left[\left(c^{+}\right)^{N}\right]_{s m_{i}}\right| 0\right\rangle \\
& =N\langle 0|\left(a_{s_{\sigma}}^{\{N\}} C_{s_{\sigma} m ; m-\mu_{i}, \mu_{i}}^{s_{\sigma}+1,1}\left[c^{N-1}\right]_{s_{\sigma}+1, m-\mu_{i}}\right. \\
& \left.+b_{s_{\sigma}}^{\{N\}} C_{s_{\sigma} m ; m-\mu_{i}, \mu_{i}}^{s_{\sigma}-1,1}\left[c^{N-1}\right]_{s_{\sigma}-1, m-\mu_{i}}\right) \\
& \cdot\left(a_{s}^{\{N\}} C_{s m_{i} ; m_{i}-\mu, \mu}^{s+1,1}\left[\left(c^{+}\right)^{N-1}\right]_{s+1, m_{i}-\mu}\right. \\
& \left.+b_{s}^{\{N\}} C_{s m_{i} ; m_{i}-\mu, \mu}^{s-1,1}\left[\left(c^{+}\right)^{N-1}\right]_{s-1, m_{i}-\mu}\right)|0\rangle \\
& =N \delta_{m-\mu_{i}, m_{i}-\mu}\left\{\left[\left(a_{s}^{\{N\}}\right)^{2} C_{s m ; m-\mu_{i}, \mu_{i}}^{s+1,1} C_{s m_{i} ; m_{i}-\mu, \mu}^{s+1,1}\right.\right. \\
& \left.+\left(b_{s}^{\{N\}}\right)^{2} C_{s m ; m-\mu_{i}, \mu_{i}}^{s-1,1} C_{s m_{i} ; m_{i}-\mu, \mu}^{s-1,1}\right] \delta_{s_{\sigma}, s} \\
& +a_{s_{\sigma}}^{\{N\}} b_{s}^{\{N\}} C_{s_{\sigma} m ; m-\mu_{i}, \mu_{i}}^{s_{\sigma}+1,1} C_{s m_{i} ; m_{i}-\mu, \mu}^{s-1,1} \delta_{s_{\sigma}, s-2} \\
& \left.+b_{s_{\sigma}}^{\{N\}} a_{s}^{\{N\}} C_{s_{\sigma} m ; m-\mu_{i}, \mu_{i}}^{s_{\sigma}-1,1} C_{s m_{i} ; m_{i}-\mu, \mu}^{s+1,1} \delta_{s_{\sigma}, s+2}\right\} .
\end{aligned}
$$

Specifically, for $s=N$, which is the case interesting for us, from (B7) and (B8), we get $a_{N}^{\{N\}}=0$, and $b_{N}^{\{N\}}=1$. Thus (B9) is simplified as

$$
\begin{aligned}
{ }_{c}\left\langle s_{\sigma},\right. & \left.m\left|c_{\mu_{i}}^{+} c_{\mu}\right| s, m_{i}\right\rangle_{c} \\
= & N \delta_{m-\mu_{i}, m_{i}-\mu}\left\{C_{N m ; m-\mu_{i}, \mu_{i}}^{N-1,1} C_{N m_{i} ; m_{i}-\mu, \mu}^{N-1,1} \delta_{\sigma, 0}\right. \\
& \left.+a_{N_{\sigma}}^{\{N\}} C_{N-2, m ; m-\mu_{i}, \mu_{i}}^{N-1,1} C_{N m_{i} ; m_{i}-\mu, \mu}^{N-1,1} \delta_{\sigma,-2}\right\} .
\end{aligned}
$$

The last term in the above equation, corresponding to the channel with final total spin $s_{\sigma}=N-2$, is suppressed by a factor $a_{N-2}^{\{N\}}=\sqrt{\frac{2 N-2}{N(2 N-3)}} \sim \frac{1}{\sqrt{N}}$. Hence, the channels between states with different values of total spin can be neglected for a large $N$.

Substituting (B10) into (B5) and neglecting the term with $a_{N-2}^{\{N\}}$, one obtains the transition matrix,

$$
\begin{aligned}
T_{m, \mu}^{m_{i}, \mu_{i}}= & N g_{0} \delta_{m-\mu_{i}, m_{i}-\mu}\left\{\delta_{m, m_{i}}\right. \\
& +C_{N, m ; m_{i}-\mu, \mu_{i}}^{N-1,1} C_{N, m_{i} ; m_{i}-\mu, \mu}^{N-1,1} .
\end{aligned}
$$

For the incident state $\left|\xi^{i}\right\rangle_{a}=\sum_{\nu=-1}^{1} \xi_{v}^{i} a_{v}^{+}|0\rangle$, the transition matrix

$$
\begin{aligned}
T_{m, \mu}^{m_{i}} & =\sum_{\nu=-1}^{1} T_{m, \mu}^{m_{i}, \nu} \xi_{\nu}^{i} \\
& =\left(\delta_{m, m_{i}}+C_{N, m ; m_{i}-\mu, \nu}^{N-1,1} C_{N, m_{i} ; m_{i}-\mu, \mu}^{N-1,1}\right) \xi_{v}^{i},
\end{aligned}
$$

where $v=m-m_{i}+\mu$, and the unimportant constant prefactor $N g_{0}$ has been dropped. The above equation is the Eq. (3) of the main text.

\section{Transition matrix for $s=N$ by the group theory}

In the subspace of $s=N$, we denote $|N, m\rangle_{c}$ as $|m\rangle_{c}$ for the sake of brevity. Under the $S O(3)$ group of transformations, the operator products $c_{v}^{+} c_{\mu}$ are transformed according to the tensor product of the two representations $3 \otimes 3^{*}$, which have the decomposition

$$
c_{\nu}^{+} c_{\mu}=-(-1)^{\mu} C_{J, n ; \nu,-\mu}^{1,1} Q_{n}^{J},
$$

where $\left\{Q_{n}^{J} \mid n=0, \pm 1, \cdots, \pm J\right\}$ is an irreducible set of operators following the $J$-irreducible representation of $S O(3)$, with $J=0,1,2$ from the direct decomposition of $3 \otimes 3^{*}$. Then, the transition matrix elements have the form

$$
{ }_{c}\left\langle m\left|c_{\nu}^{+} c_{\mu}\right| m_{i}\right\rangle_{c}=-(-1)^{\mu} C_{J, n ; \nu,-\mu}^{1,1}{ }_{c}\left\langle m\left|Q_{n}^{J}\right| m_{i}\right\rangle_{c}
$$

According to the Wigner-Echart theorem, the matrix elements of an irreducible operator in states of an irreducible representation can be written as

$$
{ }_{c}\left\langle m\left|Q_{n}^{J}\right| m_{i}\right\rangle_{c}=\frac{C_{m_{i}, n ; N, m}^{N, J}}{\sqrt{2 N+1}}{ }_{c}\left\langle N|| Q^{J} \| N\right\rangle_{c},
$$

where ${ }_{c}\left\langle N\left\|Q^{J}\right\| N\right\rangle_{c}$ are reduced matrix elements that do not depend on the magnetic quantum numbers.

By choosing three special matrix elements that can be calculated explicitly, one can obtain the reduced matrix elements. For instance, it is straight forward to calculate the matrix elements: ${ }_{c}\left\langle N\left|c_{1}^{+} c_{1}\right| N\right\rangle_{c},{ }_{c}\left\langle N\left|c_{0}^{+} c_{0}\right| N\right\rangle_{c}$, and ${ }_{c}\left\langle N\left|c_{-1}^{+} c_{-1}\right| N\right\rangle_{c}$. Substitutions of the results in (B14) lead to a group of three linear algebraic equations for the reduced matrix elements. The solutions are

$$
\begin{gathered}
{ }_{c}\left\langle N\left\|Q^{0}\right\| N\right\rangle_{c}=N \sqrt{\frac{2 N+1}{3},} \\
{ }_{c}\left\langle N\left\|Q^{1}\right\| N\right\rangle_{c}=\sqrt{\frac{N(N+1)(2 N+1)}{2}}, \\
{ }_{c}\left\langle N\left\|Q^{2}\right\| N\right\rangle_{c}=\sqrt{\frac{(2 N+3)(2 N+2)(2 N+1) N}{12(2 N-1)}} .
\end{gathered}
$$

Substitutions of the above reduced matrix elements in (B15) give the same expression as the first term of (B10). This equivalency is sufficient for the SBEC with $s=N$. Generalization of the above results, from $s=N$ to an arbitrary value of $s$, by this method is rather a tedious task.

\section{APPENDIX C: MAGNETIZATION FLUCTUATION OF THE CONDENSATE IN EARLY TIME OF SCATTERING WITH A SYMMETRIC INCIDENT ATOM BEAM}

Following Eq. (3) of the main text, the squared transition matrix has the following form:

$$
\begin{aligned}
\left|T_{m, \mu}^{m^{\prime}}\right|^{2}= & \delta_{m^{\prime}, m}\left[1+\left(C_{m-\mu, \mu}\right)^{2}\right]^{2}\left|\xi_{\mu}^{i}\right|^{2} \\
& +\delta_{m^{\prime}, m \pm 1}\left(C_{m^{\prime}-\mu, \mu \mp 1} C_{m^{\prime}-\mu, \mu}\right)^{2}\left|\xi_{\mu \mp 1}^{i}\right|^{2} \\
& \delta_{m^{\prime}, m \pm 2}\left(C_{m^{\prime}-\mu, \mu \mp 2} C_{m^{\prime}-\mu, \mu}\right)^{2}\left|\xi_{\mu \mp 2}^{i}\right|^{2}
\end{aligned}
$$

where summing over the plus and minus signs is implied, and the normalization factor

$$
\begin{aligned}
Z\left(t_{n}\right)= & \sum_{m, \mu} \lambda_{m}\left(t_{n-1}\right)\left\{\left[1+\left(C_{m-\mu, \mu}\right)^{2}\right]^{2}\left|\xi_{\mu}^{i}\right|^{2}\right. \\
& +\left(C_{m-\mu, \mu \mp 1} C_{m-\mu, \mu}\right)^{2}\left|\xi_{\mu \mp 1}^{i}\right|^{2} \\
& \left.\left(C_{m-\mu, \mu \mp 2} C_{m-\mu, \mu}\right)^{2}\left|\xi_{\mu \mp 2}^{i}\right|^{2}\right\} .
\end{aligned}
$$

The Clebsch-Gordan coefficients for coupling the spin states $|S=N-1, m\rangle$ and $|S=1, \mu\rangle$ the state $|S=N, m\rangle$ are 
known as

$$
\begin{aligned}
C_{m, \mu} \equiv & C_{N, m+\mu ; m, \mu}^{N-1,1} \\
= & \mu^{2}\left[\frac{(N+\mu m)(N+\mu m+1)}{2 N(2 N-1)}\right]^{\frac{1}{2}} \\
& +\left(1-\mu^{2}\right)\left[\frac{N^{2}-m^{2}}{N(2 N-1)}\right]^{\frac{1}{2}} .
\end{aligned}
$$

Take the magnetic quantum number per atom $x=\frac{m}{N}$ and its $k$ th moment at $t_{n}$ defined as

$$
\bar{x}_{k}\left(t_{n}\right)=\frac{1}{N^{k} Z\left(t_{n}\right)} \sum_{m} m^{k} \lambda_{m}\left(t_{n}\right)
$$

Substitutions of (4) and (C1) in (C4) give

$$
\begin{aligned}
\bar{x}_{k}\left(t_{n}\right) & \\
= & \frac{1}{N^{k} Z\left(t_{n}\right)} \sum_{m, \mu} \lambda_{m}\left(t_{n-1}\right)\left\{m^{k}\left[1+\left(C_{m-\mu, \mu}\right)^{2}\right]^{2}\left|\xi_{\mu}^{i}\right|^{2}\right. \\
& +(m \mp 1)^{k}\left(C_{m-\mu, \mu \mp 1} C_{m-\mu, \mu}\right)^{2}\left|\xi_{\mu \mp 1}^{i}\right|^{2} \\
& \left.+(m \mp 2)^{k}\left(C_{m-\mu, \mu \mp 2} C_{m-\mu, \mu}\right)^{2}\left|\xi_{\mu \mp 2}^{i}\right|^{2}\right\}
\end{aligned}
$$

and thus the summations over $m$ in (C2) and (C5) can be expressed in terms of the moments at $t_{n-1}$,

$$
\bar{x}_{k}\left(t_{n}\right)=\frac{\sum_{j=0}^{k+2} g_{k, j} \bar{x}_{j}\left(t_{n-1}\right)}{N^{k} \sum_{j=0}^{2} z_{j} \bar{x}_{j}\left(t_{n-1}\right)} .
$$

Consider the condensate having the symmetric initial spin distribution $\lambda_{m}(0)=\delta_{m, 0}$ and the incident atom state $\left|\xi^{0 y}\right\rangle_{a}=$ $\frac{1}{\sqrt{2}}\left(|-1\rangle_{a}+|1\rangle_{a}\right)$. Due to the global spin-reversal symmetry, the magnetization per atom $\bar{x}_{1}=0$, therefore, $\bar{x}_{2}=\chi$ is the fluctuation of $x$,

$$
\chi\left(t_{n}\right)=\frac{g_{2,0}+g_{2,2} \chi\left(t_{n-1}\right)+g_{2,4} \bar{x}_{4}\left(t_{n-1}\right)}{N^{2}\left[z_{0}+z_{2} \chi\left(t_{n-1}\right)\right]}
$$

Using (C3), we obtain following prefactors of $\chi\left(t_{n-1}\right)$ and $\bar{x}_{j}\left(t_{n-1}\right)$ in the right-hand side of (C7):

$$
z_{0}=-1+4 N-13 N^{2}+14 N^{3}
$$

$$
\begin{gathered}
z_{2}=3 N-7 N^{2}+6 N^{3}, \\
g_{2,0}=-4-2 N+5 N^{2}+3 N^{3}, \\
g_{2,2}=14 N-14 N^{2}-6 N^{3}-9 N^{4}+14 N^{5}, \\
g_{2,4}=10 N^{3}-11 N^{4}+6 N^{5} .
\end{gathered}
$$

For $N \gg 1, \chi\left(t_{n}\right)$ can be approximated by a continuous function of $t_{n}$ and the difference of $\chi$ between consecutive scatterings approaches the derivative

$$
\frac{d \chi\left(t_{n}\right)}{d t_{n}} \approx \chi\left(t_{n}\right)-\chi\left(t_{n-1}\right)
$$

Relations (C8)-(C13) enable us to transform (C7) into following differential equation:

$$
\frac{d \chi}{d t}=\frac{g_{2,0}+\left(g_{2,2}-N^{2} z_{0}\right) \chi+g_{2,4} \bar{x}_{4}-N^{2} z_{2} \chi^{2}}{N^{2}\left(z_{0}+z_{2} \chi\right)} .
$$

In the early time, the fluctuation is small and one can neglect cumulants of order higher than two. Since $\bar{x}_{4}=3 \chi^{2}$ then (C14) becomes

$$
\frac{d \chi}{d t}=\frac{g_{2,0}+\left(g_{2,2}-N^{2} z_{0}\right) \chi+\left(3 g_{2,4}-N^{2} z_{2}\right) \chi^{2}}{N^{2}\left(z_{0}+z_{2} \chi\right)}
$$

Making use of (C8)-(C12) and up to the leading order of $N,(\mathrm{C} 15)$ can be further approximated by

$$
\frac{d \chi}{d t}=\frac{6 \chi^{2}}{7+3 \chi} \text {. }
$$

The integration of (C16) yields

$$
\frac{7}{6 \chi\left(t_{0}\right)}-\frac{7}{6 \chi(t)}+\frac{1}{2} \ln \frac{\chi(t)}{\chi\left(t_{0}\right)}=t-t_{0} .
$$

Neglecting the small logarithmic term, one obtains

$$
\chi(t)=\frac{\chi\left(t_{0}\right)}{1-\frac{6}{7} \chi\left(t_{0}\right)\left(t-t_{0}\right)} .
$$

[1] J. Eisert, M. Friesdorf, and C. Gogolin, Nat. Phys. 11, 124 (2015).

[2] R. Islam, R. Ma, P. M. Preiss, M. E. Tai, A. Lukin, M. Rispoli, and M. Greiner, Nature (London) 528, 77 (2015).

[3] A. M. Kaufman, M. E. Tai, A. Lukin, M. Rispoli, R. Schittko, P. M. Preiss, and M. Greiner, Science 353, 794 (2016).

[4] K. X. Wei, C. Ramanathan, and P. Cappellaro, Phys. Rev. Lett. 120, 070501 (2018).

[5] J. M. Deutsch, Phys. Rev. A 43, 2046 (1991).

[6] M. Srednicki, Phys. Rev. E 50, 888 (1994).

[7] M. Rigol, V. Dunjko, and M. Olshanii, Nature (London) 452, 854 (2008).

[8] M. Brunelli, L. Fusco, R. Landig, W. Wieczorek, J. HoelscherObermaier, G. Landi, F. L. Semião, A. Ferraro, N. Kiesel,
T. Donner, G. De Chiara, and M. Paternostro, Phys. Rev. Lett. 121, 160604 (2018).

[9] D. M. Stamper-Kurn, M. R. Andrews, A. P. Chikkatur, S. Inouye, H.-J. Miesner, J. Stenger, and W. Ketterle, Phys. Rev. Lett. 80, 2027 (1998).

[10] J. Stenger, S. Inouye, D. M. Stamper-Kurn, H.-J. Miesner, A. P. Chikkatur, and W. Ketterle, Nature (London) 396, 345 (1998).

[11] D. S. Hall, M. R. Matthews, J. R. Ensher, C. E. Wieman, and E. A. Cornell, Phys. Rev. Lett. 81, 1539 (1998); 81, 4531 (1998).

[12] D. S. Hall, M. R. Matthews, C. E. Wieman, and E. A. Cornell, Phys. Rev. Lett. 81, 1543 (1998); 81, 4532 (1998).

[13] H. Schmaljohann, M. Erhard, J. Kronjäger, K. Sengstock, and K. Bongs, Appl. Phys. B: Lasers Opt. 79, 1001 (2004).

[14] M. S. Chang, Q. Qin, W. Zhang, L. You, and M. S. Chapman, Nat. Phys. 1, 111 (2005). 
[15] C. K. Law, H. Pu, and N. P. Bigelow, Phys. Rev. Lett. 81, 5257 (1998).

[16] H. Pu, C. K. Law, S. Raghavan, J. H. Eberly, and N. P. Bigelow, Phys. Rev. A 60, 1463 (1999).

[17] T.-L. Ho, Phys. Rev. Lett. 81, 742 (1998).

[18] T. Ohmi and K. Machida, J. Phys. Soc. Jpn. 67, 1822 (1998).

[19] Y. Kawaguchi and M. Ueda, Phys. Rep. 520, 253 (2012).

[20] D. M. Stamper-Kurn and M. Ueda, Rev. Mod. Phys. 85, 1191 (2013).

[21] T.-L. Ho, Phys. Rev. Lett. 92, 090402 (2004).

[22] G. T. Landi, T. Tomé, and M. J. de Oliveira, J. Phys. A 46, 395001 (2013).

[23] M. Horikoshi, M. Koashi, H. Tajima, Y. Ohashi, and M. Kuwata-Gonokami, Phys. Rev. X 7, 041004 (2017).

[24] J. P. Santos, G. T. Landi, and M. Paternostro, Phys. Rev. Lett. 118, 220601 (2017).
[25] C. D. Hamley, E. M. Bookjans, G. Behin-Aein, P. Ahmadi, and M. S. Chapman, Phys. Rev. A 79, 023401 (2009).

[26] Z. B. Li, C. G. Bao, and W. Z. Zheng, J. Phys. B: At. Mol. Opt. Phys. 51, 095301 (2018).

[27] E. V Goldstein and P. Meystre, Phys. Rev. A 59, 3896 (1999).

[28] M. Koashi and M. Ueda, Phys. Rev. Lett. 84, 1066 (2000).

[29] T. L. Ho and S. K. Yip, Phys. Rev. Lett. 84, 4031 (2000).

[30] L.-M. Duan, J. I. Cirac, and P. Zoller, Phys. Rev. A 65, 033619 (2002).

[31] C. G. Bao and Z. B. Li, Phys. Rev. A 70, 043620 (2004).

[32] A. Widera, F. Gerbier, S. Folling, T. Gericke, O. Mandel, and I. Bloch, New J. Phys. 8, 152 (2006).

[33] C. G. Bao, Front. Phys. China 1, 92 (2006)

[34] C. G. Bao and Z. B. Li, Phys. Rev. A 72, 043614 (2005) 